\title{
Action approach to cosmological perturbations: the 2nd order metric in matter dominance
}

\author{
Lotfi Boubekeur ${ }^{\mathrm{a}}$, Paolo Creminelli ${ }^{\mathrm{a}}$, \\ Jorge Noreña ${ }^{\mathrm{b}}$, and Filippo Vernizzi ${ }^{\mathrm{a}}$ \\ a Abdus Salam ICTP, Strada Costiera 11, 34014 Trieste, Italy \\ b SISSA/ISAS, Via Beirut 2, 34014 Trieste, Italy
}

\begin{abstract}
We study nonlinear cosmological perturbations during the post-inflationary evolution, using the equivalence between a perfect barotropic fluid and a derivatively coupled scalar field with Lagrangian $\left[-(\partial \phi)^{2}\right]^{(1+w) / 2 w}$. Since this Lagrangian is just a special case of $k$-inflation, this approach is analogous to the one employed in the study of non-Gaussianities from inflation. We use this method to derive the second order metric during matter dominance in the comoving gauge directly as function of the primordial inflationary perturbation $\zeta$. Going to Poisson gauge, we recover the metric previously derived in the literature.
\end{abstract}

\section{Introduction}

Recently, there has been an extraordinary improvement in the accuracy of cosmological observations, especially regarding the statistical properties and the evolution of perturbations around a FLRW Universe. In order to fully exploit the data, it has become necessary to go beyond linear perturbation theory. This is mandatory, for instance, if one wants to study primordial non-Gaussianities which, by definition, are sensitive to the non-linear interactions of the theory.

The calculation of primordial non-Gaussianities in various models is based on a perturbative expansion of the action - or of the equations of motion - of one or more scalar fields. In general, the mixing of these scalar fields with gravity cannot be neglected. This can be done by using an ADM approach which allows to write an action for the relevant degrees of freedom taking into account gravitational perturbations [1]. In this paper we want to extend this formalism to the study of cosmological perturbations after inflation, during the standard FLRW evolution.

During its evolution, the Universe is filled with cosmological fluids such as radiation or cold dark matter. At first sight a fluid is very different from a scalar field, so that it is not clear how to extend the aforementioned approach to our case. However, in most cases the cosmological fluids can be considered as perfect and barotropic. In this case, as we will review in section 2 , the dynamics of the 
fluid is exactly equivalent to the one of a derivatively coupled scalar field, playing the role of the fluid potential velocity. Using this equivalence, the usual treatment of perturbations in the primordial Universe, used when they are generated and stretched out of the Hubble radius, can be extended to their subsequent evolution in the FLRW Universe, when the modes re-enter the Hubble radius.

In this article this approach is applied to find the exact metric at second order during the matter dominated era. Our results are compatible with those obtained previously in $[2,3]$, by directly working at the level of the Einstein equations. The knowledge of the second order metric is the first step for the calculation of any observable beyond the linear approximation. This calculations are quite relevant: in the case of the CMB anisotropy the expected magnitude of a generic second order effect is comparable to the sensitivity of the forthcoming Planck satellite.

As we will see, the calculation of the second order metric will closely parallel the one of the three-point function of scalar and tensor perturbations generated during inflation. In particular, we will make use of the action calculated for this purpose for $k$-inflation $[4,5]$, expanded up to third order. In this context, it is natural to use the comoving curvature perturbation $\zeta$ as the variable describing the scalar perturbations, the same which is commonly employed for the quantization of scalar fluctuations during inflation. This variable is also well-known to be nonlinearly conserved on super-Hubble scales independently of the details of the cosmological evolution [6]. For this reason it is the natural variable to set the initial conditions for the post-inflationary dynamics. Therefore, an advantage of our approach is that the dynamics of perturbations is described by the same variable and the same approach, from its generation at early times to the late times observations.

As a warm-up, we start with linear perturbation theory in section 3 . Then, in section 4, we extend our calculation to second order for scalar and tensor modes, assuming that primordial gravitational waves are negligible. In this way we obtain the full second order metric in the comoving gauge including tensor modes generated by scalars. In section 5 we check our results with the ones obtained in Poisson gauge $[2,3]$ after a suitable second order gauge transformation. Conclusions are drawn in section 6.

\section{A fluid as a scalar field}

In cosmology, when dissipative phenomena are negligible, the energy content of the Universe can be approximated as a sum of perfect fluids. A perfect fluid is defined to have a stress-energy tensor of the form

$$
T_{\mu \nu}=(\rho+p) u_{\mu} u_{\nu}+p g_{\mu \nu}
$$

where $\rho$ and $p$ are the energy density and the pressure, while $u^{\mu}$ is the fluid 4-velocity. Cosmological fluids are usually taken to be irrotational. This assumption is justified by the absence of vorticity in the initial conditions set by inflation and by the fact that vorticity is diluted by the expansion of the Universe. The fluids are also taken to be barotropic, i.e., their pressure is a function of the energy density only, $p=p(\rho)$. Under these conditions, each fluid is characterized by a single scalar function, so that it is not surprising that its dynamics can be described in terms of a scalar field.

Indeed, let us consider a derivatively coupled scalar $\phi$ in Minkowski spacetime with Lagrangian density $^{1}$

$$
\mathcal{L}=P(X), \quad X \equiv-\partial_{\mu} \phi \partial^{\mu} \phi .
$$

\footnotetext{
${ }^{1}$ For Lagrangian approaches which describe also vorticous motion and non-barotropic perfect fluids see
} 
Varying the action yields the equation of motion

$$
\partial_{\mu}\left[P^{\prime}(X) \partial^{\mu} \phi\right]=0
$$

The stress-energy tensor of this field is given by

$$
T_{\mu \nu}=2 P^{\prime}(X) \partial_{\mu} \phi \partial_{\nu} \phi+P(X) g_{\mu \nu}
$$

which is of the perfect fluid form (1) if we identify

$$
\rho=2 P^{\prime} X-P, \quad p=P, \quad u_{\mu}=\frac{\partial_{\mu} \phi}{\sqrt{X}} .
$$

The perfect fluid interpretation makes sense only if $\partial_{\mu} \phi$ is everywhere timelike and future directed. Projecting the conservation equation of the stress-energy tensor of a fluid, $\partial_{\mu} T_{\nu}^{\mu}=0$, along and orthogonal to the fluid flux yields the equation of conservation of energy and the Euler equation. In the case of the stress-energy tensor (4), the Euler equation is a trivial identity, while the conservation of the energy is equivalent to the equation of motion (3).

The equation of motion (3) can be interpreted as the conservation of the current

$$
J^{\mu}=2 \sqrt{X} P^{\prime}(X) \cdot u^{\mu} .
$$

This conservation is a consequence (by Nöther theorem) of the invariance of the action under shift of $\phi: \phi \rightarrow \phi+$ const. From the fluid point of view this current describes the conserved particle density flux $J^{\mu} \equiv n u^{\mu}$, where $n$ is the number particle density. Therefore one can identify $n=2 \sqrt{X} P^{\prime}(X)$. This yields a physical interpretation of the norm of $\partial_{\mu} \phi: \sqrt{X}=(\rho+P) / n$ is the so called specific inertial mass $[7,8]$. It is also straightforward to verify that

$$
\sqrt{X}=\frac{d \rho}{d n}
$$

so that $\sqrt{X}$ acts as a sort of conjugate variable with respect to $n[9]$.

It is well known that for a perfect fluid the entropy per particle is conserved along the fluid flow. This can be checked using the continuity equation $\partial_{\mu} J^{\mu}=0$, i.e. eq. (3), and the energy conservation $u^{\mu} \partial_{\nu} T_{\mu}^{\nu}=0$ [12]. Furthermore, as we have discussed, our approach describes a barotropic fluid which implies that the entropy per particle is everywhere constant. In other words, the Lagrangian (2) can only describe mechanical excitations of the fluid. It cannot take into account dissipative irreversible processes like heat conduction or viscosity.

We will be interested in studying perturbations around a homogeneous configuration. In Minkowski spacetime $\phi=c t$ is a solution of the equation of motion for any $c$. Different values of $c$ describe different unperturbed values of the energy density $\rho\left(c^{2}\right)$. The dynamics of fluctuations around this background can be studied by expanding the Lagrangian using $\phi=c t+\delta \phi(t, \vec{x})$, where $\delta \phi$ describes the compressional mode of the fluid. At second order we obtain

$$
\mathcal{L}=P^{\prime}\left(c^{2}\right)\left[\dot{\delta \phi}^{2}-(\nabla \delta \phi)^{2}\right]+2 P^{\prime \prime}\left(c^{2}\right) c^{2} \dot{\delta \phi}^{2}
$$

$[7,8,9]$. 
From this expression one sees that the speed of sound of the excitations is given by

$$
c_{s}^{2}=\left.\frac{P^{\prime}(X)}{P^{\prime}(X)+2 X P^{\prime \prime}(X)}\right|_{X=c^{2}}
$$

which, as expected, is the usual adiabatic speed of sound in a barotropic fluid,

$$
c_{s}^{2}=\left.\frac{p^{\prime}(X)}{\rho^{\prime}(X)}\right|_{X=c^{2}}=\frac{d p}{d \rho} .
$$

Note that although the Lagrangian (2) is Lorentz invariant, this symmetry is spontaneously broken by the vacuum $\phi=c t$. For this reason the speed of perturbations will in general differ from the speed of light $c_{s}^{2}=1$.

A standard case of barotropic fluid is given by a linear equation of state $p=w \rho$ with $w=$ const. From the first two equalities in eq. (5) one deduces (see for instance [10, 11])

$$
P=X^{\frac{1+w}{2 w}}, \quad w \neq 0
$$

up to a proportionality constant which is irrelevant for the classical theory. In this case, the speed of sound in eq. (10) reduces to $c_{s}^{2}=w$. As an example, one can consider a radiation fluid with equation of state $w=1 / 3$. In this case the Lagrangian (11) reduces to

$$
\mathcal{L}=X^{2}=\left(-\partial_{\mu} \phi \partial^{\mu} \phi\right)^{2}
$$

The inclusion of gravity is completely straightforward. The equation of motion (3) becomes

$$
\partial_{\mu}\left[\sqrt{-g} P^{\prime}(X) \partial^{\mu} \phi\right]=0 \text {. }
$$

In an expanding FLRW background, the homogeneous solution satisfies now

$$
n=P^{\prime} \dot{\phi} \propto a^{-3} .
$$

Indeed, for a constant $w$ this is the standard redshift of the energy density,

$$
\rho \propto \dot{\phi}^{\frac{1+w}{w}} \propto a^{-3(1+w)} .
$$

To summarize, we are able to describe the dynamics of one or more fluids coupled with gravity, within a Lagrangian formalism. This can be employed to study cosmological perturbations in the presence of barotropic fluids. Note that the Lagrangian (2) is a particular case of the so called $k$-essence/ $k$-inflation scenarios $[13,14]$. For this class of Lagrangians, cosmological perturbations have been extensively studied starting from [11] and more recently extended to second $[4,5]$ and third order [15] to study the non-Gaussianities produced during inflation. In this paper these calculations can be reinterpreted as describing the nonlinearities of a fluid.

In the following we will be interested in the study of dark matter perturbations, which would correspond to the dust case $w=0$. Obviously the zero pressure limit must be taken with care, as from eq. (5) the Lagrangian strictly vanishes for a pressureless fluid. 


\section{Background and linear dynamics}

Following the discussion of the last section, the dynamics of a perfect fluid coupled with gravity can be described by the action

$$
S=\frac{1}{2} \int \mathrm{d}^{4} x \sqrt{-g}[R+2 P(X)],
$$

where we chose units such that $M_{P}^{-2} \equiv 8 \pi G_{N}=1$. In particular we are interested in the case $p=w \rho$ with constant $w$, when $P$ is given by eq. (11).

We assume a flat FLRW metric $d s^{2}=-d t^{2}+a^{2}(t) d \vec{x}^{2}$. Friedmann equations read

$$
\begin{aligned}
H^{2} & =\frac{1}{3}\left(2 X P^{\prime}-P\right), \\
2 \dot{H} & +3 H^{2}=-P,
\end{aligned}
$$

where $H \equiv \dot{a} / a$ is the Hubble expansion parameter. It is useful to define

$$
\epsilon \equiv-\frac{\dot{H}}{H^{2}}=\frac{3}{2}(1+w) \text {. }
$$

Note that, although the notation is inspired by the inflationary case, we are not assuming that $\epsilon$ is small. ${ }^{2}$

Following Maldacena [1], the study of perturbations can be done using the ADM splitting of the metric [16],

$$
d s^{2}=-N^{2} d t^{2}+h_{i j}\left(d x^{i}+N^{i} d t\right)\left(d x^{j}+N^{j} d t\right) .
$$

The action (16) becomes

$$
S=\frac{1}{2} \int \mathrm{d} t \mathrm{~d}^{3} x \sqrt{h}\left[N\left(R^{(3)}+2 P\right)+N^{-1}\left(E_{i j} E^{i j}-E^{2}\right)\right],
$$

where $R^{(3)}$ is the curvature scalar computed from $h_{i j}, E \equiv E_{i}^{i}$ and

$$
E_{i j} \equiv \frac{1}{2}\left(\dot{h}_{i j}-\nabla_{i} N_{j}-\nabla_{j} N_{i}\right) \text {. }
$$

The covariant derivatives $\nabla_{i}$ are with respect to the 3 -metric $h_{i j}$ and all roman indeces $i, j, \ldots$ are raised and lowered with this metric.

The action (16) describes 3 dynamical degrees of freedom: one scalar mode for the fluid excitations and the 2 tensor helicities of the gravity waves. In the ADM formalism, these degrees of freedom are contained in the scalar field $\phi$ and in the 3-metric $h_{i j}$, while the lapse $N$ and the shift $N_{i}$ are not dynamical. Following [1] we choose the gauge

$$
\begin{gathered}
\delta \phi=0, \quad h_{i j}=a^{2} e^{2 \zeta} \hat{h}_{i j}, \quad \hat{h}_{i j}=\delta_{i j}+\gamma_{i j}+\frac{1}{2} \gamma_{i l} \gamma_{l j}+\ldots, \\
\operatorname{det} \hat{h}=1, \quad \gamma_{i i}=0, \quad \partial_{i} \gamma_{i j}=0,
\end{gathered}
$$

\footnotetext{
${ }^{2}$ To simplify the comparison with the literature we give here the parameters used in refs. $[4,5]$ for the $w=$ const case:

$$
\eta \equiv \frac{\dot{\epsilon}}{\epsilon H}=0, \quad u \equiv 1-\frac{1}{c_{s}^{2}}=1-\frac{1}{w}, \quad s \equiv \frac{1}{H} \frac{\dot{c_{s}}}{c_{s}}=0
$$

and

$$
\Sigma=X P^{\prime}+2 X^{2} P^{\prime \prime}=\frac{H^{2} \epsilon}{w}, \quad \lambda=X^{2} P^{\prime \prime}+\frac{2}{3} X^{3} P^{\prime \prime \prime}=-\frac{H^{2} \epsilon}{6 w}\left(1-\frac{1}{w}\right) .
$$


where $\zeta$ describes the scalar mode and $\gamma$ the tensor ones. This gauge is comoving in the sense that the fluid 4-velocity is everywhere orthogonal to the equal time surfaces ${ }^{3}$.

As the action does not contain time derivatives of $N$ and $N_{i}$, these variables act as Lagrange multipliers, i.e. their equations of motion are algebraic constraints. These equations are the momentum and Hamiltonian constraints of Einstein equations:

$$
\begin{gathered}
\nabla_{i}\left[N^{-1}\left(E_{j}^{i}-\delta_{j}^{i} E\right)\right]=0, \\
R^{(3)}+2 P-4 X P^{\prime}-\frac{1}{N^{2}}\left(E_{i j} E^{i j}-E^{2}\right)=0 .
\end{gathered}
$$

To warm up let us calculate the action for the scalar mode $\zeta$ at second order, in order to find the linear equation of motion and the first order metric. As there is no mixing between $\zeta$ and $\gamma$ at second order in the action, in this section we can set $\gamma=0$. To compute the second order action, we need to solve the constraints $(26,27)$ and plug their solution for $N$ and $N_{i}$ back into the action (23). This needs to be done at first order only, since the second-order solutions for $N$ and $N^{i}$ will multiply $\delta \mathcal{L} / \delta N$ or $\delta \mathcal{L} / \delta N^{i}$ at zeroth order, which vanish on the background [1]. To solve the momentum constraint (26) we decompose $N_{i}$ as $N_{i} \equiv \partial_{i} \psi+N_{T i}$ where $\partial_{i} N_{T i}=0$. By defining $N=1+\delta N$, one finds, at first order,

$$
\delta N=\frac{\dot{\zeta}}{H}, \quad N_{T i}=0 .
$$

Furthermore, one can find $\psi$ at first order by solving the energy constraint ${ }^{4}(27)$,

$$
\psi=-\frac{\zeta}{H}+\frac{a^{2} \epsilon}{w} \partial^{-2} \dot{\zeta}
$$

Note that the last term of the previous equation contains $w$ at the denominator so that one is not allowed to take the limit $w \rightarrow 0$ at this stage.

Substituting the solutions $(28,29)$ into the action $(23)$ one obtains, after some integration by parts, the second order action for $\zeta$,

$$
S_{2}=\int \mathrm{d} t \mathrm{~d}^{3} x a^{3} \frac{\epsilon}{w}\left[\dot{\zeta}^{2}-\frac{w}{a^{2}}(\partial \zeta)^{2}\right] .
$$

The equation of motion derived from this action is thus

$$
\ddot{\zeta}+3 H \dot{\zeta}-\frac{w}{a^{2}} \partial^{2} \zeta=0 \text {. }
$$

We are interested in the limit $w \rightarrow 0$. Setting $w=0$, the growing solution of eq. (31) is simply a constant, $\zeta=\zeta_{0}$, where $\zeta_{0}$ is the perturbation generated during inflation, which remains constant on super-Hubble scales independently of the equation of state ${ }^{5}$. However, to find the metric we need

\footnotetext{
${ }^{3}$ We follow the notation of [1] using $\zeta$ to denote the curvature perturbation in the comoving gauge. Often this quantity is instead called $\mathcal{R}$ reserving $\zeta$ to denote the curvature perturbation in the uniform density gauge.

${ }^{4}$ Here and in the following $\partial^{2} \equiv \partial_{i} \partial_{i}$ and $\partial^{-2}$ is its inverse.

${ }^{5}$ For a generic equation of state $p(\rho)$ the action for $\zeta$ is

$$
S=\int \mathrm{d} t \mathrm{~d}^{3} x a^{3} \frac{\epsilon}{c_{s}^{2}}\left[\dot{\zeta}^{2}-\frac{c_{s}^{2}}{a^{2}}(\partial \zeta)^{2}\right],
$$


to plug the solution for $\zeta$ into eqs. $(28,29)$ and for $\dot{\zeta}$ in eq. (29) we need to keep the leading order correction in $w$ in eq. (31).

The general solution of this equation in Fourier space can be written as a linear combination of Bessel functions,

$$
\zeta_{\vec{k}}(\tau)=C_{1} \tau^{-\nu} J_{\nu}(k \tau \sqrt{w})+C_{2} \tau^{-\nu} Y_{\nu}(k \tau \sqrt{w}), \quad \nu=\frac{3(1-w)}{2(1+3 w)},
$$

where we have introduced the conformal time $\tau=\int d t / a$. The growing mode of this solution goes as

$$
\zeta=\zeta_{0}+\frac{2 w}{5 a^{2} H^{2}} \partial^{2} \zeta_{0}+\mathcal{O}\left(w^{2}\right),
$$

where $\zeta_{0}$ is the asymptotic value of $\zeta$ on super-Hubble scales. The time derivative of (35) is

$$
\dot{\zeta}=\frac{2 w}{5 a^{2} H} \partial^{2} \zeta_{0}+\mathcal{O}\left(w^{2}\right)
$$

This can be plugged into eqs. $(28,29)$ which yield, at lowest order in $w \rightarrow 0$,

$$
\delta N=0, \quad \psi=-\frac{2}{5} \frac{\zeta_{0}}{H} .
$$

The metric at first order in perturbations is thus given by

$$
d s^{2}=-d t^{2}-\frac{4}{5 H} \partial_{i} \zeta_{0} d t d x^{i}+a^{2}\left(1+2 \zeta_{0}\right) d \vec{x}^{2} .
$$

Note that under a coordinate transformation

$$
t \rightarrow t+\frac{2 \zeta_{0}}{5 H}, \quad x^{i} \rightarrow x^{i}
$$

this metric takes the Newtonian gauge form

$$
d s^{2}=-(1+2 \Phi) d t^{2}+a^{2}(1-2 \Psi) d \vec{x}^{2},
$$

with

$$
\Phi=\Psi=-\frac{3}{5} \zeta_{0}
$$

which is the well known linear relation between the Newtonian potential and $\zeta_{0}$ in matter dominance.

where $c_{s}^{2}$ is given by eq. (9). This gives the following equation of motion

$$
\ddot{\zeta}+\dot{\zeta} \frac{d}{d t} \ln \left(a^{3} \frac{\epsilon}{c_{s}^{2}}\right)-\frac{c_{s}^{2}}{a^{2}} \partial^{2} \zeta=0 .
$$

From this equation we see that $\zeta$ is constant on large scales, $k / a H \ll 1$, independently of the energy content. This is not true, for instance, for the Newtonian potential. 


\section{Second order perturbations}

\subsection{Scalar perturbations}

We are interested in deriving the third order action for $\zeta$. In order to do that we follow the same procedure we did at linear order and initially set $\gamma=0$ everywhere. Tensor modes generated at second order by scalar perturbations will be considered in the next section. To derive the third order action we only need the solutions to the constraint equations at linear order, i.e. eqs. $(28,29)$. This is because the second and third order solutions would multiply the first and zeroth order constraint equations, respectively [1]. We will be interested in the second order solutions of the constraint equations only later, when we will derive the explicit expression of the metric at second order.

It is straightforward to derive

$$
\begin{aligned}
E_{i j} E^{i j}-E^{2}= & -6(H+\dot{\zeta})^{2}+4 \frac{e^{-2 \zeta}}{a^{2}}(H+\dot{\zeta})\left(\partial_{i} N_{i}+\partial_{i} \zeta N_{i}\right) \\
& +\frac{e^{-4 \zeta}}{a^{4}}\left[\frac{1}{4}\left(\partial_{i} N_{j}+\partial_{j} N_{i}\right)^{2}-\left(\partial_{i} N_{j}+\partial_{j} N_{i}\right)\left(N_{i} \partial_{j} \zeta+N_{j} \partial_{i} \zeta\right)-\left(\partial_{i} N_{i}\right)^{2}\right]
\end{aligned}
$$

and

$$
R^{(3)}=-2 \frac{e^{-2 \zeta}}{a^{2}}\left[2 \partial^{2} \zeta+(\partial \zeta)^{2}\right]
$$

Expanding $P(X)$ with $X=\dot{\phi}^{2} / N^{2}$ and using the first equality in (28) one obtains [4]

$$
\begin{aligned}
P(X)= & P+P^{\prime}\left(-2 \frac{\dot{\zeta}}{H}+3 \frac{\dot{\zeta}^{2}}{H^{2}}-2 \delta N_{2}-4 \frac{\dot{\zeta}^{3}}{H^{3}}+6 \delta N_{2} \frac{\dot{\zeta}}{H}\right) X \\
& +\frac{1}{2} P^{\prime \prime}\left(4 \frac{\dot{\zeta}^{2}}{H^{2}}+8 \delta N_{2} \frac{\dot{\zeta}}{H}-12 \frac{\dot{\zeta}^{3}}{H^{3}}\right) X^{2}-\frac{4}{3} P^{\prime \prime \prime} \frac{\dot{\zeta}^{3}}{H^{3}} X^{3},
\end{aligned}
$$

where, on the right hand side, $X, P$ and its derivatives are evaluated at zeroth order. The lapse has been split as $N=1+\delta N_{1}+\delta N_{2}$, where $\delta N_{1}=\dot{\zeta} / H$ is the first order contribution, eq. (28), while $\delta N_{2}$ is the second order one. As we discussed, terms containing $\delta N_{2}$ will not appear in the action; however they will be important to derive the metric.

Plugging the previous expressions into the action and using the solutions to the constraint equation (28) one obtains, after several integrations by parts, the third order action for $\zeta$,

$$
\begin{aligned}
S_{3}= & \int \mathrm{d} t \mathrm{~d}^{3} x a^{3}\left[\frac{3 \epsilon}{w} \dot{\zeta}^{2} \zeta-\frac{\epsilon}{3 H w}\left(2+\frac{1}{w}\right) \dot{\zeta}^{3}-\frac{\epsilon}{a^{2}}(\partial \zeta)^{2} \zeta\right. \\
& \left.-\frac{2}{a^{4}} \partial_{i} \zeta \partial_{i} \psi \partial^{2} \psi+\frac{1}{2 a^{4}}\left(\partial_{i} \partial_{j} \psi \partial_{i} \partial_{j} \psi-\left(\partial^{2} \psi\right)^{2}\right)\left(3 \zeta-\frac{\dot{\zeta}}{H}\right)\right]
\end{aligned}
$$

where $\psi$ is given by eq. (29),

$$
\psi=-\frac{\zeta}{H}+\frac{a^{2} \epsilon}{w} \partial^{-2} \dot{\zeta}
$$

This action describes the cosmological scalar perturbations of a perfect fluid with constant equation of state $w$. Notice that it is just a particular case of the general action derived in refs. [4, 5] in the context of generalized inflationary models, although it describes here a quite different physical system. 
The action is simplified by a field redefinition, $\zeta_{n}=\zeta-f(\zeta)$, where [5]

$$
\begin{aligned}
f(\zeta)= & \frac{1}{w H} \zeta \dot{\zeta}+\frac{1}{4 a^{2} H^{2}}\left[-\left(\partial_{i} \zeta\right)^{2}+\partial^{-2} \partial_{i} \partial_{j}\left(\partial_{i} \zeta \partial_{j} \zeta\right)\right] \\
& +\frac{\epsilon}{2 H^{2} w}\left[\partial_{i} \zeta \partial_{i} \partial^{-2} \dot{\zeta}-\partial^{-2} \partial_{i} \partial_{j}\left(\partial_{i} \zeta \partial_{j} \partial^{-2} \dot{\zeta}\right)\right] .
\end{aligned}
$$

As $f$ is quadratic in $\zeta$, the field redefinition does not change the second order action. Note that the function $f(\zeta)$ contains either spatial gradients or time derivatives of $\zeta$. Thus, for any constant $w$, the field redefinition (47) vanishes on super-Hubble scales, so that in this regime $\zeta$ and $\zeta_{n}$ coincide. In terms of $\zeta_{n}$ the action reads

$$
\begin{aligned}
S_{3}= & \int \mathrm{d} t \mathrm{~d}^{3} x a^{3} \frac{\epsilon}{w}\left[\frac{2}{3}\left(\frac{1}{w}-1\right) \frac{\dot{\zeta}_{n}^{3}}{H}+\frac{3}{2}\left(3-\frac{1}{w}\right) \zeta_{n} \dot{\zeta}_{n}^{2}+\frac{1}{2 a^{2}}(5+w) \zeta_{n}\left(\partial_{i} \zeta_{n}\right)^{2}\right. \\
& \left.-\left(2-\frac{\epsilon}{2}\right) \frac{\epsilon}{w} \dot{\zeta}_{n} \partial_{i} \zeta_{n} \partial_{i} \partial^{-2} \dot{\zeta}_{n}+\frac{\epsilon^{2}}{4 w} \partial^{2} \zeta_{n}\left(\partial_{i} \partial^{-2} \dot{\zeta}_{n}\right)^{2}\right] .
\end{aligned}
$$

We are interested in a dust fluid $w=0$. The equation of motion at second order derived from this action can be solved perturbatively by plugging the first order solutions $\zeta=\zeta_{n}=\zeta_{0}$ into the second order terms. At lowest order in $w \rightarrow 0$ one finds

$$
\ddot{\zeta}_{n}+3 H \dot{\zeta}_{n}=\frac{1}{a^{2}} F_{0}
$$

where

$$
F_{0}=-\zeta_{0} \partial^{2} \zeta_{0}-\frac{5}{16}\left(\partial_{i} \zeta_{0}\right)^{2}-\frac{3}{8} \partial_{i} \partial^{-2}\left(\partial^{2} \zeta_{0} \partial_{i} \zeta_{0}\right)
$$

The right hand side of eq. (49) is negligible at very early times, when all the modes are well out of the Hubble radius, $k / a H \ll 1$, so that $\zeta_{n}=$ const is a solution. As in this regime $\zeta$ and $\zeta_{n}$ coincide as discussed, this corresponds to the well-known fact that $\zeta$ is conserved on super-Hubble scales, even nonlinearly. In this regime the Fourier modes of $\zeta$ are decoupled and equal to the initial condition set by inflation, $\zeta=\zeta_{0}$ for $k / a H \ll 1$. Solving the equation above and going back to $\zeta$ using (47) yields

$$
\zeta=\zeta_{0}-\frac{1}{5 a^{2} H^{2}} \partial^{-2} \partial_{i} \partial_{j}\left(\partial_{i} \zeta_{0} \partial_{j} \zeta_{0}\right)
$$

This expression gives $\zeta$ at second order during matter dominance, as a function of its initial condition $\zeta_{0}$. We postpone its discussion to the end of this section and we proceed to conclude the calculation of the second order metric: we need to solve for $N$ and $N_{i}$ at second order.

As at first order, to solve for the whole metric we need to keep terms of order $w$ in the second order evolution equation which now reads

$$
\ddot{\zeta}_{n}+3 H \dot{\zeta}_{n}-\frac{w}{a^{2}} \partial^{2} \zeta_{n}=\frac{1}{a^{2}}\left(F_{0}+w F_{1}\right)+\frac{w}{a^{4} H^{2}} G,
$$

where

$$
\begin{aligned}
F_{1}= & -5 \zeta_{0} \partial^{2} \zeta_{0}+\frac{1}{8}\left(\partial_{i} \zeta_{0}\right)^{2}+\frac{3}{4} \partial_{i} \partial^{-2}\left(\partial^{2} \zeta_{0} \partial_{i} \zeta_{0}\right) \\
G= & -\frac{21}{25}\left(\partial^{2} \zeta_{0}\right)^{2}-\frac{2}{5} \zeta_{0} \partial^{4} \zeta_{0}+\frac{1}{20} \partial_{i} \zeta_{0} \partial_{i} \partial^{2} \zeta_{0} \\
& +\frac{9}{200} \partial^{2}\left(\partial_{i} \zeta_{0}\right)^{2}-\frac{3}{50} \partial_{i} \partial^{-2}\left(\partial_{i} \zeta_{0} \partial^{4} \zeta_{0}\right) .
\end{aligned}
$$


To obtain the $w$ corrections to the right-hand side we used the linear evolution of $\zeta$ at order $w^{2}$ :

$$
\dot{\zeta}=\frac{2 w}{5 a^{2} H} \partial^{2} \zeta_{0}-\frac{6 w^{2}}{25 a^{2} H} \partial^{2} \zeta_{0}+\frac{4 w^{2}}{35 a^{4} H^{3}} \partial^{4} \zeta_{0}+\mathcal{O}\left(w^{3}\right)
$$

Solving eq. (52) and writing the solution in terms of $\zeta$ using the field redefinition (47) one finds

$$
\begin{aligned}
& \zeta=\zeta_{0}-\frac{1}{5 a^{2} H^{2}} \partial^{-2} \partial_{i} \partial_{j}\left(\partial_{i} \zeta_{0} \partial_{j} \zeta_{0}\right) \\
&+\frac{w}{5 a^{2} H^{2}}[ 2 \partial^{2} \zeta_{0}-4 \zeta_{0} \partial^{2} \zeta_{0}+\frac{14}{5}\left(\partial_{i} \zeta_{0}\right)^{2}+\frac{18}{5} \partial_{i} \partial^{-2}\left(\partial^{2} \zeta_{0} \partial_{i} \zeta_{0}\right) \\
&-\frac{1}{7 a^{2} H^{2}}\left(\frac{27}{20}\left(\partial^{2} \zeta_{0}\right)^{2}+\frac{51}{10} \partial_{i} \zeta_{0} \partial_{i} \partial^{2} \zeta_{0}+\frac{4}{5}\left(\partial_{i} \partial_{j} \zeta_{0}\right)^{2}\right. \\
&\left.\left.+\frac{1}{2} \partial_{i} \partial^{-2}\left(\partial_{i} \zeta_{0} \partial^{4} \zeta_{0}\right)+\frac{1}{5} \partial^{-2} \partial_{i}\left(\partial_{i} \partial_{j} \zeta_{0} \partial_{j} \partial^{2} \zeta_{0}\right)\right)\right]+\mathcal{O}\left(w^{2}\right)
\end{aligned}
$$

Now that we have a solution for $\zeta$ including $\mathcal{O}(w)$ corrections, we can proceed to solve the constraints to get the final second order metric. The momentum constraint is a vector equation; taking its transverse part with the projector $\delta_{i j}-\partial_{i} \partial_{j} / \partial^{2}$ we obtain an equation for the transverse part of the shift vector $N_{T i}\left(\partial_{i} N_{T i}=0\right)$,

$$
N_{T i}=-\frac{4}{5} \frac{1}{H} \partial^{-2}\left[\partial_{i} \zeta_{0} \partial^{2} \zeta_{0}-\partial^{-2} \partial_{i} \partial_{k}\left(\partial_{k} \zeta_{0} \partial^{2} \zeta_{0}\right)\right] .
$$

Notice that the transverse part of the shift vanished at first order, see eq. (28). The longitudinal part of the momentum constraint gives an equation for the lapse function at second order,

$$
\begin{aligned}
\delta N_{2} & =\frac{w}{5 a^{2} H^{2}}\left[\left(\partial \zeta_{0}\right)^{2}-4 \zeta_{0} \partial^{2} \zeta_{0}\right] \\
& -\frac{2 w}{175 a^{4} H^{4}}\left[3\left(\partial^{2} \zeta_{0}\right)^{2}+14 \partial_{i} \zeta_{0} \partial_{i} \partial^{2} \zeta_{0}+4\left(\partial_{i} \partial_{j} \zeta_{0}\right)^{2}\right]+\mathcal{O}\left(w^{2}\right) .
\end{aligned}
$$

Although the lapse perturbation vanishes in the limit $w \rightarrow 0$, its expression at order $w$ is necessary to solve the energy constraint, similarly to what happened at first order. This gives the second order correction to the shift function

$$
\begin{aligned}
\psi_{2}=\frac{1}{5 H} \partial^{-2} & {\left[\left(\partial_{i} \zeta_{0}\right)^{2}-3 \partial^{-2} \partial_{i} \partial_{j}\left(\partial_{i} \zeta_{0} \partial_{j} \zeta_{0}\right)\right.} \\
& \left.+\frac{4}{5 a^{2} H^{2}}\left(\frac{3}{7}\left(\partial^{2} \zeta_{0}\right)^{2}+\partial_{i} \zeta_{0} \partial_{i} \partial^{2} \zeta_{0}+\frac{4}{7}\left(\partial_{i} \partial_{j} \zeta_{0}\right)^{2}\right)\right] .
\end{aligned}
$$

Plugging in the ADM metric (22) the first order results (28), (29) and the second order ones (56), (57), (58) and (59), we finally obtain the second order metric for $w=0$,

$$
\begin{aligned}
g_{00}= & -1+\frac{4}{25 a^{2} H^{2}}\left(\partial_{i} \zeta_{0}\right)^{2} \\
g_{0 i}= & -\frac{1}{5 H} \partial_{i}\left[2 \zeta_{0}-\partial^{-2}\left(\partial_{j} \zeta_{0}\right)^{2}+3 \partial^{-4} \partial_{j} \partial_{k}\left(\partial_{j} \zeta_{0} \partial_{k} \zeta_{0}\right)\right. \\
& \left.\quad-\frac{4}{5 a^{2} H^{2}} \partial^{-2}\left(\frac{3}{7}\left(\partial^{2} \zeta_{0}\right)^{2}+\partial_{i} \zeta_{0} \partial_{i} \partial^{2} \zeta_{0}+\frac{4}{7}\left(\partial_{i} \partial_{j} \zeta_{0}\right)^{2}\right)\right] \\
& -\frac{4}{5} \frac{1}{H} \partial^{-2}\left[\partial_{i} \zeta_{0} \partial^{2} \zeta_{0}-\partial^{-2} \partial_{i} \partial_{k}\left(\partial_{k} \zeta_{0} \partial^{2} \zeta_{0}\right)\right], \\
g_{i j}= & a^{2}\left[1+2 \zeta_{0}+2 \zeta_{0}^{2}-\frac{2}{5 a^{2} H^{2}} \partial^{-2} \partial_{k} \partial_{l}\left(\partial_{k} \zeta_{0} \partial_{l} \zeta_{0}\right)\right] \delta_{i j}+a^{2} \gamma_{i j} .
\end{aligned}
$$


Before moving to the calculation of the tensor contribution $\gamma$, let us discuss eq. (51) which describes the second order evolution of $\zeta$. In Fourier space this equation reads

$$
\zeta_{\vec{k}}=\zeta_{0 \vec{k}}+\frac{1}{5 a^{2} H^{2}} \int \frac{d^{3} q}{(2 \pi)^{3}} \frac{(\vec{k} \cdot \vec{q})\left(k^{2}-\vec{k} \cdot \vec{q}\right)}{k^{2}} \zeta_{0 \vec{q}} \zeta_{0 \vec{k}-\vec{q}}
$$

It is important to stress that the kernel inside the integral does not vanish in the limit $k \rightarrow 0$. Surprisingly, this implies that a very long wavelength mode well outside the Hubble radius evolves in the presence of short wavelength perturbations: $\zeta$ is not conserved on super-Hubble scales as its second order contribution in the matter dominated era grows like the scale factor $a \propto 1 / a^{2} H^{2}$. However, this is not in contrast with the literature on the conservation of $\zeta[6]$, as $\zeta$ is conserved when all the modes are out of the Hubble radius, so that the second term on the right hand side of eqs. (51) and (63) can be neglected.

The fact that $\zeta$ is not conserved in our case is not so surprising after all. Indeed, at nonlinear order one can define an infinite set of variables which coincide with $\zeta$, and which are thus conserved, when all the gradients can be neglected, i.e. out of the Hubble radius. However, when sub-Hubble modes are considered, if one of these variables remains constant, the others will not in general. In fact, one can trivially define a variable which is conserved on all scales, i.e.,

$$
\tilde{\zeta}=\zeta+\frac{1}{5 a^{2} H^{2}} \partial^{-2} \partial_{i} \partial_{j}\left(\partial_{i} \zeta \partial_{j} \zeta\right)
$$

even though this has not a particular geometrical meaning and we do not expect it to remain conserved when a different equation of state is considered. In order to discuss the physical significance of the variation of $\zeta$ one needs to compute at second order the relationship with a measurable quantity, like Cosmic Microwave Background temperature fluctuations. This goes beyond the scope of this article.

\subsection{Gravitational waves}

So far we did not discuss gravitational waves. At linear order tensor and scalar modes are decoupled, so that gravitational waves can be completely neglected in the limit where the primordial contribution generated by inflation is very small. Beyond the linear approximation scalar and tensor modes mix, so that it is not consistent to set $\gamma=0$. In the following we are going to assume that the amplitude of primordial tensor modes is very small: gravitational waves will only be generated by the scalar modes through couplings of the form $\gamma \zeta \zeta$.

To study the generation of gravitational waves we need the quadratic action for $\gamma$ and the cubic terms of the form $\gamma \zeta \zeta$. The constraint equations will not enter neither in the derivation of the action nor in obtaining the expression of the second order metric. Indeed $\gamma$ does not appear at first order in the constraint equations and quadratic terms are negligible as $\gamma=\mathcal{O}\left(\zeta^{2}\right)$.

Let us start with the quadratic action for $\gamma$. Making use of the expressions

$$
E_{i j} E^{i j}-E^{2} \supset \frac{1}{4} \dot{\gamma}_{i j} \dot{\gamma}_{i j}
$$

and

$$
R^{(3)} \supset \frac{1}{a^{2}}\left(\gamma_{i j} \partial^{2} \gamma_{i j}+\frac{3}{4}\left(\partial_{i} \gamma_{j k}\right)^{2}-\frac{1}{2} \partial_{i} \gamma_{k j} \partial_{k} \gamma_{i j}\right),
$$


after integration by parts this yields

$$
S_{\gamma \gamma}=\frac{1}{8} \int \mathrm{d} t \mathrm{~d}^{3} x a^{3}\left(\dot{\gamma}_{i j} \dot{\gamma}_{i j}-\frac{1}{a^{2}} \partial_{k} \gamma_{i j} \partial_{k} \gamma_{i j}\right)
$$

For the $\zeta \zeta \gamma$ terms we use the expressions

$$
R^{(3)} \supset \frac{4}{a^{2}} \gamma_{i j} \partial_{i} \partial_{j} \zeta-\frac{10}{a^{2}} \gamma_{i j} \zeta \partial_{i} \partial_{j} \zeta
$$

and

$$
e^{3 \zeta} N^{-1} a^{3}\left(E_{i j} E^{i j}-E^{2}\right) \supset-a \dot{\gamma}_{i j}\left(3 \zeta-\frac{\dot{\zeta}}{H}\right) \partial_{i} \partial_{j} \psi+\frac{1}{a} \partial_{k} \gamma_{i j} \partial_{i} \partial_{j} \psi \partial_{k} \psi,
$$

and we obtain

$$
\begin{aligned}
S_{\gamma \zeta \zeta}=\int \mathrm{d} t \mathrm{~d}^{3} x a^{3} & {\left[-\frac{2}{H a^{2}} \gamma_{i j} \partial_{i} \dot{\zeta} \partial_{j} \zeta-\frac{1}{a^{2}} \gamma_{i j} \partial_{i} \zeta \partial_{j} \zeta\right.} \\
& \left.-\frac{1}{2 a^{2}}\left(3 \zeta-\frac{\dot{\zeta}}{H}\right) \dot{\gamma}_{i j} \partial_{i} \partial_{j} \psi+\frac{1}{2 a^{4}} \partial_{l} \gamma_{i j} \partial_{i} \partial_{j} \psi \partial_{l} \psi\right] .
\end{aligned}
$$

This part of the action is the same as the one derived by Maldacena [1] for a scalar field with standard kinetic term. This is not surprising as these couplings do not depend on $P(X)$ in $(23)$.

The equation of motion for $\gamma$ can be obtained by varying the actions (67) and (70) with respect to $\gamma_{i j}$. Here there are no subtleties in the limit $w \rightarrow 0$ so that one can drop terms containing $\dot{\zeta}$. Notice that $\gamma_{i j}$ is constrained to satisfy the transverse and traceless conditions $\partial_{i} \gamma_{i j}=0$ and $\gamma_{i i}=0$. As such, the $\gamma$ 's in the action above must be understood as projected by the transverse and traceless projector, i.e.,

$$
P_{i j k l}^{\mathrm{TT}}=\frac{1}{2}\left(P_{i k} P_{j l}+P_{j k} P_{i l}-P_{i j} P_{k l}\right)
$$

where $P_{i j}$ is a symmetric transverse projector given by

$$
P_{i j} \equiv \delta_{i j}-\frac{\partial_{i} \partial_{j}}{\partial^{2}}
$$

Thus, the evolution equation reads

$$
\ddot{\gamma}_{i j}+3 H \dot{\gamma}_{i j}-\frac{\partial^{2}}{a^{2}} \gamma_{i j}=P_{i j k l}^{\mathrm{TT}}\left[\frac{2}{a^{2}} \partial_{k} \zeta_{0} \partial_{l} \zeta_{0}+\frac{4}{25 a^{4} H^{2}} \partial^{2}\left(\partial_{k} \zeta_{0} \partial_{l} \zeta_{0}\right)\right],
$$

where we have simplified the term in the square braket on the right hand side, taking into account that we are interested only in its transverse component. The solution of this equation, with the appropriate initial condition $\gamma_{i j}=0$, reads

$$
\gamma_{i j}=-\frac{4}{5}\left[9\left(\frac{1}{3}-\frac{j_{1}(k \tau)}{k \tau}\right) \partial^{-2}+\frac{1}{5 a^{2} H^{2}}\right] P_{i j k l}^{\mathrm{TT}}\left(\partial_{k} \zeta_{0} \partial_{l} \zeta_{0}\right)
$$

where the spherical Bessel function $j_{1}(x)$ can be written in terms of trigonometric functions as $j_{1}(x)=\sin (x) / x^{2}-\cos (x) / x$. 


\section{Transforming to Poisson gauge}

In this section we want to write our second order metric in conformal Poisson gauge, i.e. in the form

$$
\mathrm{d} s^{2}=a^{2}(\tau)\left\{-\left(1+2 \Phi_{P}\right) \mathrm{d} \tau^{2}+2 \omega_{P}^{\perp} d \tau d x^{i}+\left[\left(1-2 \Psi_{P}\right) \delta_{i j}+\gamma_{P i j}\right] \mathrm{d} x^{i} \mathrm{~d} x^{j}\right\},
$$

where $\omega_{P}{ }_{i}$ is transverse, $\partial_{i} \omega_{P}{ }_{i}=0$, and $\gamma_{P}$ is transverse and traceless. Going to this gauge will enable us to compare our results with those obtained in $[2,3]$.

For the second order gauge transformation we use the notation of [2] (apart from exchanging the name of the scalar potentials $\Phi$ and $\Psi$ ) and we write our metric as

$$
\mathrm{d} s^{2}=a^{2}(\tau)\left\{-\left(1+2 \Phi_{\zeta}\right) \mathrm{d} \tau^{2}+2\left(\omega_{\zeta i}^{\perp}+\partial_{i} \omega_{\zeta}\right) \mathrm{d} \tau \mathrm{d} x^{i}+\left[\left(1-2 \Psi_{\zeta}\right) \delta_{i j}+\gamma_{i j}\right] \mathrm{d} x^{i} \mathrm{~d} x^{j}\right\}
$$

where

$$
\begin{aligned}
\Phi_{\zeta}= & -\frac{2}{25 a^{2} H^{2}}\left(\partial_{i} \zeta_{0}\right)^{2} \\
\omega_{\zeta}= & -\frac{2}{5 a H} \zeta_{0}+\frac{1}{5 a H} \partial^{-2}\left[\left(\partial_{i} \zeta_{0}\right)^{2}-3 \partial^{-2} \partial_{i} \partial_{j}\left(\partial_{i} \zeta_{0} \partial_{j} \zeta_{0}\right)\right] \\
& +\frac{4}{25 a^{3} H^{3}} \partial^{-2}\left[\frac{3}{7}\left(\partial^{2} \zeta_{0}\right)^{2}+\partial_{i} \zeta_{0} \partial_{i} \partial^{2} \zeta_{0}+\frac{4}{7}\left(\partial_{i} \partial_{j} \zeta_{0}\right)^{2}\right], \\
\omega_{\zeta i}^{\perp}= & -\frac{4}{5 a H} \partial^{-2}\left[\partial_{i} \zeta_{0} \partial^{2} \zeta_{0}-\partial^{-2} \partial_{i} \partial_{j}\left(\partial_{j} \zeta_{0} \partial^{2} \zeta_{0}\right)\right], \\
\Psi_{\zeta}= & -\zeta_{0}-\zeta_{0}^{2}+\frac{1}{5 a^{2} H^{2}} \partial^{-2} \partial_{i} \partial_{j}\left(\partial_{i} \zeta_{0} \partial_{j} \zeta_{0}\right),
\end{aligned}
$$

and $\gamma_{i j}$ is given by eq. (74). The gauge transformation up to second order is, in conformal coordinates $x^{\mu}=\left(\tau, x^{i}\right)$,

$$
x^{\mu} \rightarrow x^{\mu}-\xi_{1}^{\mu}-\xi_{2}^{\mu}+\frac{1}{2} \xi_{1}^{\nu} \xi_{1, \nu}^{\mu}
$$

where at each order $\xi^{0}=\alpha$ and $\xi^{i}=\partial^{i} \beta+d^{i}$ with the vector $d^{i}$ transverse, $\partial_{i} d_{i}=0$. The first order piece is fixed by eq. (39), i.e. $\alpha_{1}=-2 \zeta_{0} /(5 a H)$ and $\beta_{1}=0=d_{1 i}$. The parameters of the gauge transformation at second order can be obtained from the second order components of the metric

$$
\omega_{P}^{\perp}{ }_{i}+\partial_{i} \omega_{P}=\omega_{\zeta i}^{\perp}+\partial_{i} \omega_{\zeta}+\frac{\alpha_{1}}{2} \partial_{i}\left[2\left(\alpha_{1}^{\prime}+2 a H \alpha_{1}\right)-\alpha_{1}^{\prime}-4 a H \alpha_{1}\right]-\frac{1}{2} \alpha_{1}^{\prime} \partial_{i} \alpha_{1}-\partial_{i} \alpha_{2}+\partial_{i} \beta_{2}^{\prime}+d_{2}^{\prime}{ }_{i},
$$

and

$$
\gamma_{P i j}=\gamma_{i j}+\partial_{i} \alpha_{1} \partial_{j} \alpha_{1}-\frac{1}{3} \delta_{i j}\left(\partial_{k} \alpha_{1}\right)^{2}+2\left(\partial_{i} \partial_{j}-\frac{1}{3} \delta_{i j} \partial^{2}\right) \beta_{2}+\partial_{i} d_{2 j}+\partial_{j} d_{2 i} .
$$

In the previous equations we used that $\omega_{\zeta}=-2 \zeta_{0} /(5 a H)=\alpha_{1}$ at first order. Imposing that $\gamma_{P}$ is transverse $\left(\chi_{P}^{\|}=\chi_{P}^{\perp}=0\right.$ in the notation of [2]) allows to solve for $\beta_{2}$ and $d_{2 i}$ :

$$
\begin{aligned}
\beta_{2} & =-\frac{3}{25 a^{2} H^{2}} \partial^{-2}\left[\partial^{-2} \partial_{i} \partial_{j}\left(\partial_{i} \zeta_{0} \partial_{j} \zeta_{0}\right)-\frac{1}{3}\left(\partial_{i} \zeta_{0}\right)^{2}\right] \\
d_{2 i} & =-\frac{4}{25 a^{2} H^{2}} \partial^{-2}\left[\partial^{2} \zeta_{0} \partial_{i} \zeta_{0}-\partial^{-2} \partial_{i} \partial_{j}\left(\partial^{2} \zeta_{0} \partial_{j} \zeta_{0}\right)\right] .
\end{aligned}
$$

Imposing $\omega_{P}=0$ in eq. (82) gives

$$
\alpha_{1}+\alpha_{2}=\omega_{\zeta}+\beta_{2}^{\prime}
$$


At second order, the other components of the metric transform as [2]

$$
\begin{aligned}
& \Phi_{P}=\Phi_{\zeta}+\frac{\alpha_{1}}{2}\left(\alpha_{1}^{\prime \prime}+5 a H \alpha_{1}^{\prime}+\frac{3}{2} a^{2} H^{2} \alpha_{1}\right)+\alpha_{1}^{\prime 2}+\alpha_{2}^{\prime}+a H \alpha_{2} \\
& \Psi_{P}=\Psi_{\zeta}+\frac{\alpha_{1}}{2}\left[-\frac{3}{2} a^{2} H^{2} \alpha_{1}-a H \alpha_{1}^{\prime}+2\left(\Psi_{\zeta}^{\prime}+2 a H \Psi_{\zeta}\right)\right]-\frac{1}{6}\left(\partial_{i} \alpha_{1}\right)^{2}-a H \alpha_{2}-\frac{1}{3} \partial^{2} \beta_{2},
\end{aligned}
$$

which, with the conditions (84), (85) and (86) above, give

$$
\begin{aligned}
\Phi_{P}= & -\frac{3}{5} \zeta_{0}+\frac{9}{25}\left[\zeta_{0}^{2}+\partial^{-2}\left(\partial_{j} \zeta_{0}\right)^{2}-3 \partial^{-4} \partial_{i} \partial_{j}\left(\partial_{i} \zeta_{0} \partial_{j} \zeta_{0}\right)\right] \\
& +\frac{6}{175 a^{2} H^{2}} \partial^{-2}\left[2\left(\partial_{i} \partial_{j} \zeta_{0}\right)^{2}+5\left(\partial^{2} \zeta_{0}\right)^{2}+7 \partial_{i} \zeta_{0} \partial_{i} \partial^{2} \zeta_{0}\right], \\
\Psi_{P}= & -\frac{3}{5} \zeta_{0}-\frac{9}{25}\left[\zeta_{0}^{2}+\frac{2}{3} \partial^{-2}\left(\partial_{i} \zeta_{0}\right)^{2}-2 \partial^{-4} \partial_{i} \partial_{j}\left(\partial_{i} \zeta_{0} \partial_{j} \zeta_{0}\right)\right] \\
& +\frac{6}{175 a^{2} H^{2}} \partial^{-2}\left[2\left(\partial_{i} \partial_{j} \zeta_{0}\right)^{2}+5\left(\partial^{2} \zeta_{0}\right)^{2}+7 \partial_{i} \zeta_{0} \partial_{i} \partial^{2} \zeta_{0}\right], \\
\omega_{P}^{\perp}= & -\frac{24}{25 a H} \partial^{-2}\left[\partial^{2} \zeta_{0} \partial_{i} \zeta_{0}-\partial^{-2} \partial_{i} \partial_{j}\left(\partial^{2} \zeta_{0} \partial_{j} \zeta_{0}\right)\right], \\
\gamma_{P i j}= & -\frac{36}{5}\left(\frac{1}{3}-\frac{j_{1}(k \tau)}{k \tau}\right) \partial^{-2} P_{i j k l}^{\mathrm{TT}}\left(\partial_{k} \zeta_{0} \partial_{l} \zeta_{0}\right) .
\end{aligned}
$$

The transverse traceless projector above can be expanded to give

$$
P_{i j k l}^{\mathrm{TT}}\left(\partial_{k} \zeta_{0} \partial_{l} \zeta_{0}\right)=-\partial^{-2}\left[\partial^{2} \Theta_{0} \delta_{i j}+\partial_{i} \partial_{j} \Theta_{0}+2\left(\partial^{2} \zeta_{0} \partial_{i} \partial_{j} \zeta_{0}-\partial_{i} \partial_{k} \zeta_{0} \partial_{j} \partial_{k} \zeta_{0}\right)\right]
$$

with

$$
\Theta_{0}=-\frac{1}{2} \partial^{-2}\left[\left(\partial^{2} \zeta_{0}\right)^{2}-\left(\partial_{i} \partial_{j} \zeta_{0}\right)^{2}\right] .
$$

This result can be compared with ref. [3] by taking into account that $\zeta_{0}=-(5 / 3) \Phi$ at linear order and on super-Hubble scales. The resulting metrics coincide up to a typo in eq. (A.29) of $[3] .^{6}$ Notice that in order to perform the calculation in Poisson gauge, one has to supplement the second order equations obtained in [2] by the proper second order matching with the initial condition provided by $\zeta$ [3]. In our calculation this matching is already taken into account by the fact that we are always using the conserved quantity $\zeta$.

\section{Discussion}

In this article we have developed a new approach to nonlinear cosmological perturbations, based on the equivalence between cosmological perfect fluids and derivatively coupled scalar fields. In this approach, perturbations can be studied using an ADM perturbative expansion of the scalar field action, similarly to what is done for the study of non-Gaussianities during inflation.

Using this method, we have calculated the second order metric during matter domination in the comoving gauge (25), where the scalar fluctuations are described by the comoving curvature

\footnotetext{
${ }^{6}$ To obtain the correct result the right hand side of eq. (A.29) of [3] should be multiplied by a factor -2 . Note that in their notation a factor of $1 / 2$ multiplies all the second order contributions, i.e., $\omega_{i} \equiv \omega_{1 i}+\frac{1}{2} \omega_{2 i}$ while in our notation there is no factor of $1 / 2$.
} 
perturbation $\zeta$. The metric, which is the main result of the paper, is given by

$$
\begin{aligned}
g_{00}= & -1+\frac{4}{25 a^{2} H^{2}}\left(\partial_{i} \zeta_{0}\right)^{2} \\
g_{0 i}= & -\frac{1}{5 H} \partial_{i}\left[2 \zeta_{0}-\partial^{-2}\left(\partial_{j} \zeta_{0}\right)^{2}+3 \partial^{-4} \partial_{j} \partial_{k}\left(\partial_{j} \zeta_{0} \partial_{k} \zeta_{0}\right)\right. \\
& \left.\quad-\frac{4}{5 a^{2} H^{2}} \partial^{-2}\left(\frac{3}{7}\left(\partial^{2} \zeta_{0}\right)^{2}+\partial_{i} \zeta_{0} \partial_{i} \partial^{2} \zeta_{0}+\frac{4}{7}\left(\partial_{i} \partial_{j} \zeta_{0}\right)^{2}\right)\right] \\
& -\frac{4}{5} \frac{1}{H} \partial^{-2}\left[\partial_{i} \zeta_{0} \partial^{2} \zeta_{0}-\partial^{-2} \partial_{i} \partial_{j}\left(\partial_{j} \zeta_{0} \partial^{2} \zeta_{0}\right)\right] \\
g_{i j}= & a^{2} \exp [2 \zeta(t)] \delta_{i j}+a^{2} \gamma_{i j},
\end{aligned}
$$

where

$$
\zeta(t)=\zeta_{0}-\frac{1}{5 a^{2} H^{2}} \partial^{-2} \partial_{k} \partial_{l}\left(\partial_{k} \zeta_{0} \partial_{l} \zeta_{0}\right)
$$

$\zeta_{0}$ is the perturbation generated during inflation, conserved on super-Hubble scales, and where $\gamma_{i j}$ is a traceless transverse tensor, given by

$$
\gamma_{i j}=-\frac{4}{5}\left[9\left(\frac{1}{3}-\frac{j_{1}(k \tau)}{k \tau}\right) \partial^{-2}+\frac{1}{5 a^{2} H^{2}}\right] P_{i j k l}^{\mathrm{TT}}\left(\partial_{k} \zeta_{0} \partial_{l} \zeta_{0}\right)
$$

Note that at early times, when all the modes are out of the Hubble radius so that we can neglect gradients, the above metric is just

$$
d s^{2}=-d t^{2}+a^{2}(t) e^{2 \zeta_{0}(\vec{x})} d \vec{x}^{2}
$$

This is the form of the metric in comoving gauge after all the modes have left the Hubble radius during inflation. This metric is known to remain the same during the subsequent evolution of the Universe until the modes re-enter the Hubble radius. This shows that our metric nicely matches the initial conditions set by inflation.

As shown by eq. (98) above, we have found that $\zeta$ is not conserved at second order during the matter dominated era. Indeed, super-Hubble modes can evolve under the presence of short subHubble perturbations. However, we have concluded that a discussion on the physical significance of this variation cannot be done without the computation of the relation between $\zeta$ and observable quantities, which is beyond the scope of this article.

Our method can be generalized to compute the metric during a cosmological era dominated by a fluid with constant equation of state $w \neq 0$, like during radiation dominance, by choosing the appropriate $w$ in the linear evolution equation for $\zeta$, eq. (31), and in the third order action (48). The only difference is that in this case $\zeta$ is not constant inside the Hubble (see eq. (31)), so that the second order solution will involve an integral over conformal time. Furthermore, one can generalize our method to study the evolution of perturbations in a Universe filled with several coupled fluids, by introducing a correspondent number of derivatively coupled scalar fields in the action, with possible couplings between them. As a future study, this method could be used to study the generation of gravitational waves from second order perturbations during radiation and matter dominated era $[17,18]$. 


\section{Acknowledgments}

We thank Francis Bernardeau, Chris Clarkson, Guido D'Amico, Ruth Durrer, David Langlois, Sabino Matarrese, Alberto Nicolis, Cyril Pitrou, Riccardo Rattazzi and Jean-Philippe Uzan for useful discussions.

\section{References}

[1] J. M. Maldacena, "Non-Gaussian features of primordial fluctuations in single field inflationary models," JHEP 0305 (2003) 013 [arXiv:astro-ph/0210603].

[2] S. Matarrese, S. Mollerach and M. Bruni, "Second-order perturbations of the Einstein-de Sitter universe," Phys. Rev. D 58 (1998) 043504 [arXiv:astro-ph/9707278].

[3] N. Bartolo, S. Matarrese and A. Riotto, "The Full Second-Order Radiation Transfer Function for LargeScale CMB Anisotropies," JCAP 0605, 010 (2006) [arXiv:astro-ph/0512481].

[4] D. Seery and J. E. Lidsey, "Primordial non-gaussianities in single field inflation," JCAP 0506, 003 (2005) [arXiv:astro-ph/0503692].

[5] X. Chen, M. x. Huang, S. Kachru and G. Shiu, "Observational signatures and non-Gaussianities of general single field inflation," JCAP 0701, 002 (2007) [arXiv:hep-th/0605045].

[6] D. S. Salopek and J. R. Bond, "Nonlinear evolution of long wavelength metric fluctuations in inflationary models," Phys. Rev. D 42, 3936 (1990); D. H. Lyth, K. A. Malik and M. Sasaki, "A general proof of the conservation of the curvature perturbation," JCAP 0505, 004 (2005) [arXiv:astro-ph/0411220]; D. Langlois and F. Vernizzi, "Conserved non-linear quantities in cosmology," Phys. Rev. D 72, 103501 (2005) [arXiv:astro-ph/0509078].

[7] A. H. Taub, "General Relativistic Variational Principle for Perfect Fluids," Phys. Rev. 94, 1468 (1954).

[8] B. F. Schutz, "Perfect Fluids in General Relativity: Velocity Potentials and a Variational Principle," Phys. Rev. D 2, 2762 (1970).

[9] S. Dubovsky, T. Gregoire, A. Nicolis and R. Rattazzi, "Null energy condition and superluminal propagation," JHEP 0603 (2006) 025 [arXiv:hep-th/0512260].

[10] S. Matarrese, "On The Classical And Quantum Irrotational Motions Of A Relativistic Perfect Fluid. 1. Classical Theory," Proc. Roy. Soc. Lond. A 401, 53 (1985).

[11] J. Garriga and V. F. Mukhanov, "Perturbations in k-inflation," Phys. Lett. B 458, 219 (1999) [arXiv:hepth/9904176].

[12] S. Weinberg, Gravitation and Cosmology, John Wiley and Sons 1972.

[13] C. Armendariz-Picon, T. Damour and V. F. Mukhanov, "k-inflation," Phys. Lett. B 458, 209 (1999) [arXiv:hep-th/9904075].

[14] C. Armendariz-Picon, V. F. Mukhanov and P. J. Steinhardt, "Essentials of k-essence," Phys. Rev. D 63, 103510 (2001) [arXiv:astro-ph/0006373].

[15] F. Arroja and K. Koyama, "Non-gaussianity from the trispectrum in general single field inflation," Phys. Rev. D 77, 083517 (2008) [arXiv:0802.1167 [hep-th]].

[16] R. Arnowitt, S. Deser and C. W. Misner, "The dynamics of general relativity," arXiv:gr-qc/0405109.

[17] K. N. Ananda, C. Clarkson and D. Wands, Phys. Rev. D 75, 123518 (2007) [arXiv:gr-qc/0612013].

[18] D. Baumann, P. J. Steinhardt, K. Takahashi and K. Ichiki, Phys. Rev. D 76, 084019 (2007) [arXiv:hepth/0703290]. 